\title{
EFFECT OF TEMPORAL LOBECTOMY FOR EPILEPSY ON LANGUAGE DEVELOPMENT
}

Language development was examined in 24 children (mean age 11 years; range 5.815.7 years) with AED intractable epilepsy before and 6, 12, and 24 months after anterior temporal lobectomy, in a study by the Dutch Collaborative Epilepsy Surgery Programme (DuCESP) at University Medical Center Utrecht, The Netherlands. Before surgery, the mean language delay varied from 1.7 years to 3.5 years and after surgery, language development was slower than normal, except for receptive syntax (execution of oral commands). Productive lexicon (oral response to visually presented objects) was slowed when surgery and language mediation were both in the left hemisphere. (de Koning T, Versnel H, Jennekens-Schinkel A, et al. Language development before and after temporal surgery in children with intractable epilepsy. Epilepsia Nov 2009;50(11):2408-2419). (Respond: Huib Versnel, FO2.504, Department of Otorhinolaryngology, Rudolf Magnus Institute of Neuroscience, University Medical Center Utrecht, PO Box 85500, 3508 GA Utrecht, The Netherlands. E-mail: h.versnel@umcutrecht.nl).

COMMENT. Intractable temporal lobe epilepsy is a risk factor for delay in language development, and surgery does not result in improved language. Some language components are slowed when surgery and language mediation both involve the left hemisphere. Temporal lobectomy of the non-dominant hemisphere is not expected to affect language development.

\section{PAROXYSMAL DISORDERS}

\section{FRONTAL-TEMPORAL BRAIN VOLUMES IN ABSENCE EPILEPSY}

Fronto-temporal brain volumes and their association with clinical and psychological variables in children, aged 7.5-11.8 years, with childhood absence epilepsy (CAE) were compared to age and gender-matched children without epilepsy. The CAE group $(n=26)$ had significantly smaller gray matter volumes of the left orbital frontal gyrus and both temporal lobes vs controls $(\mathrm{n}=37)$. Brain volumes in $\mathrm{CAE}$ children were related to age, gender, ethnicity, and pregnancy complications but not to seizure, IQ, and psychopathology variables. In controls without epilepsy, brain volumes were related to IQ. (Caplan R, Levitt J, Siddarth P, et al. et al. Frontal and temporal volumes in childhood absence epilepsy. Epilepsia Nov 2009;50(11):2466-2472). (Respond: Rochelle Caplan MD, Semel Institute for Neuroscience and Human Behavior, 760 Westwood Plaza, Los Angeles, CA 90024. E-mail: rcaplan@ucla.edu).

COMMENT. This study supports the theory of an orbital frontal focus for CAE spikes (Holmes et al Epilepsia 2004;45:1568-1579), and a symptomatic localization related cause for CAE, Lack of an association between orbital frontal gyrus gray matter volumes and seizure variables suggests that an underlying neuropathology, not seizures, affects brain development in children with CAE. 